\title{
PROPUESTAS PARA IMPLEMENTAR UN SISTEMA DE INFORMACIÓN EN LA EDUCACIÓN SUPERIOR CHILENA
}

\section{Escenario actual de los sistemas de información en la educación superior chilena}

La expansión del sistema de educación superior (ES) que experimentó el país en las dos últimas décadas se caracterizó por un crecimiento inorgánico, generado por los aumentos de cobertura de matrícula, oferta educativa y expansión territorial, a la vez que por la carencia de elementos que dieran cuenta de la calidad de esta evolución.

La preocupación por la calidad de las instituciones y sus programas residía principalmente en la Comisión Nacional de Acreditación (CNAP) y en la Comisión Nacional de Acreditación de Postgrado (CONAP). Luego, el tema adquirió mayor relevancia con la discusión de la ley sobre el Sistema Nacional de Aseguramiento de la Calidad de la educación superior (SNAC).

Un insumo esencial a la hora de precisar la calidad de una institución o programa es la información que sobre ellos se dispone. Ésta debe servir tanto para dar a conocer al público la calidad de la oferta como para que los organismos e instituciones tengan un instrumento que dé cuenta de los niveles de calidad alcanzados en sus principales actividades ${ }^{\mathrm{ii}}$.

ii Proyecto de Ley del SNAC (artículo $1^{\circ}$, letra a), donde se establece que es función del sistema "de información, que tendrá por objeto la identificación, recolección y difusión de los antecedentes necesarios para la gestión del sistema, la gestión institucional y la información pública". 
Junto con esta mayor preocupación por la calidad desde un punto de vista del sistema en general, la competitividad hizo que las instituciones fueran paulatinamente generando y perfeccionando estrategias y planes de desarrollo que, para su aplicación, requieren disponer de buena información, tanto de sus procesos internos como de los de otras instituciones. De esta forma, los mecanismos de acreditación han sido adoptados por las universidades como la plataforma que se necesitaba para medir su evolución y la competitividad y efectividad de su gestión, sirviendo, en muchos casos, para instaurar procesos y registros de datos inexistentes hasta ese momento.

Para disponer de tales datos, las instituciones han desarrollado sus propios sistemas de información con adecuaciones o salidas especiales para responder a las múltiples solicitudes externas. El incentivo para usar las definiciones establecidas en los requerimientos externos de información es doble, por cuanto hay un ahorro de esfuerzos, al no tener que desarrollar trabajos paralelos, y, la más importante, permite la comparación fácil con otras instituciones.

Por lo general, los sistemas de información de las instituciones se alimentan de sistemas específicos (contabilidad, personal, registros académicos, entre otros) que, en muchos casos, se encuentran en plataformas y estructuras de datos diferentes. La tendencia actual es facilitar la integración de los sistemas, para lo cual se recurre a plataformas comunes (paquetes de software de clase mundial), programación a medida o generando las interfases necesarias para la comunicación de los subsistemas.

En esta estructura de múltiples sistemas es cada vez más común encontrar en las universidades unidades a cargo de generar la información estratégica, a la vez que responder las solicitudes externas y recoger los datos para el benchmarking. Estas unidades no producen la información al interior de las universidades, pero sí la procesan para la gestión y le dan sentido a la que se encuentra fraccionada. 
De esta misma manera, subiendo a un nivel más agregado en el sistema de ES, se encuentran varios organismos que, por sus funciones, interactúan con las instituciones en áreas específicas y otros que se encargan de darle sentido a esos subsistemas, especialmente para apoyar al público en sus decisiones. Algunos de estos son: el Ministerio de Educación (Mineduc), su División de Educación Superior (Divesup), Mecesup, la reciente Secretaría Ejecutiva a cargo de administrar los nuevos créditos de la Ley 20.02, la Comisión Nacional de Investigación Científica y Tecnológica (CONICYT), el Consejo Superior de Educación (CSE) y la Comisión Nacional de Acreditación (CNAP). Estos organismos replican nuevamente el esquema de trabajo de las universidades con sistemas específicos y entes que centralizan información de distintos ámbitos.

La propuesta central de este artículo reconoce esta situación y su diseño contempla la tarea descentralizada de subsistemas especializados de información, pero con una coordinación muy fuerte para disponer de un Sistema de Información para la Educación Superior (SIES) que cuente con vías únicas de alimentación desde las instituciones y cuyos datos sean compartidos entre sus integrantes para maximizar su uso y difusión.

\section{¿Cómo implementar el SIES?}

Para llegar a implantar este sistema es necesario, primero, entender que el SIES no sirve, si se piensa como un software o como un modelo sobre el que ya no se actúa una vez implementado. El SIES debe dar cuenta de un sistema inherentemente dinámico; por ello necesita ser adecuado en forma constante a nuevas necesidades que se generen sobre él.

Por otra parte, si bien el SIES estará sustentado en una ley, lo mejor para su operación es que su funcionamiento se base en una relación armoniosa entre solicitantes y proveedores de información; entre el costo de producir un dato y su real aporte al sistema. 
Para implementar el SIES se proponen seguir cinco pasos o conjuntos de tareas, los cuales forman un ciclo que debe estar ejecutándose continuamente para mantener un sistema actualizado, mejorado y adaptado a las necesidades emergentes:

1. Establecer objetivos, metas y alcances del sistema. Para ello se requiere identificar las entidades que conforman el sistema, su participación en el SIES, las fronteras del sistema y los flujos de información entre las entidades.

2. Determinar las áreas de información que requieren ser cubiertas por el sistema.

3. Establecer un conjunto de variables que permitan reflejar de mejor manera cada una de las áreas temáticas que abordará el SIES. Para cada una de ellas se debe establecer una definición exacta y un ente encargado de solicitarlo, determinar quién debe generar o producir la información, con qué periodicidad, cómo validarla y una estrategia para su obtención.

4. Definir cuáles serán los canales de difusión y entrega de la información. Se sugiere múltiples canales conforme a los diferentes entes a los que se quiere llegar, bajo la modalidad de un mismo dato entregado por distintas vías.

5. Instaurar procesos continuos de mejoras y retroalimentación con los usuarios y las entidades participantes. Debe haber una constante revisión de los aspectos tecnológicos y operativos, tales como seguridad, traspaso de datos, almacenamiento, plataformas, formatos, restricciones, accesos, entre otros.

\section{Aplicación de las etapas}

Para que este trabajo no sea exclusivamente teórico, se propone una serie de actividades de implementación.

\section{Objetivos y alcances del sistema}

1.1. Objetivo: Disponer de un Sistema de Información de la Educación Superior (SIES) de acuerdo con las necesidades del 
Sistema Nacional de Acreditación y de la comunidad, que sea viable, operativamente funcional y sustentable en el tiempo.

Una buena opción para disponer de un sistema con estas características es que el SIES integre varios subsistemas coordinados, vinculados en línea y cuya información sea homologada y normalizada.

\subsection{Las entidades que conforman el sistema:}

- Mineduc (sus diversas unidades: Registro Institucional, Aportes, Donaciones, Ayudas Estudiantiles, Mecesup y otros).

- CNAP, CSE.

- Instituciones de educación superior (universidades, institutos profesionales, centros de formación técnica).

- Agencias acreditadoras de programas.

- CONICYT.

- Usuarios: estudiantes, padres, investigadores, medios de comunicación, otros organismos (ministerios, Superintendencia de Valores, Congreso, embajadas, entidades financieras, Registro Civil, entre otros).

\subsection{Principales roles de los participantes}

Ministerio de Educación

El Mineduc debería generar la columna vertebral de la estructura de datos del SIES, con la cual debiera conectarse el resto de las entidades. Para ello sería conveniente un Departamento de Gestión de Información, al interior de la División de Educación Superior, que fuera el centro y motor del funcionamiento del SIES. Su tarea sería coordinar el trabajo de los otros entes integrantes del sistema. Por lo tanto, asumiría la responsabilidad final del SIES.

Otra de sus tareas sería liderar la construcción gradual del sistema. Dada la magnitud del trabajo es indispensable establecer una hoja de ruta. 


\section{Las agencias acreditadoras}

Un elemento vital de esta propuesta tiene que ver con la modificación de no entregar una gran cantidad de datos al público, sino sólo los relevantes. Estos debieran surgir a partir de los resultados de los procesos de acreditación y ser distribuidos por el SIES.

Hasta antes de la acreditación se ofrecía una amplia gama de datos e indicadores con la intención de bosquejar la calidad de la oferta de cada institución. Este gran conjunto de insumos (infraestructura, laboratorios, computadores, bibliotecas, entre otros) se obtenía de consultas directas a las instituciones y se caracterizaba por un grado de respuesta parcial y de dudosa comparación entre entidades. Ahora, con los resultados de los procesos de acreditación -con un alto grado de validez-, se puede identificar tanto las fortalezas como debilidades de cada programa o institución acreditada.

Operando dentro del SIES, las agencias acreditadoras cumplirían una función indirecta de validación de los datos, ya que ellas recogerían esta información pública al inicio de sus procesos de acreditación y, luego, en el transcurso y fin del proceso, podrían constatar su certeza.

Tareas comunes de los organismos ${ }^{\mathrm{iii}}$ : captura y recopilación de datos

Esta propuesta se define por solicitar pocos datos, pero de buena calidad: no pedir información procesada (indicadores, por ejemplo) sino datos elementales de los cuales se tenga certeza y que sean un aporte directo a las necesidades del público.

En esta línea se propone realizar solicitudes a las instituciones de ES espaciadas durante el año, conforme a las particularidades de cada tema (admisión, titulación, estados financieros, etc.).

iii Mayor detalle en: Peñafiel, Antonio, Un Sistema de Información para la Educación Superior Chilena, Documento de trabajo CPU, No 1/2006, págs. 24 y 25. 
Además, algunas peticiones realizarlas entre periodos mayores de tiempo (informes bianuales, trianuales, por ejemplo).

Se debería elaborar un calendario consensuado de funcionamiento. Es indispensable conocer con anticipación las fechas y contenidos de los procesos de recolección de datos y la forma y momento en que se difundirán.

Es indispensable un acuerdo entre los organismos para que cada dato tenga un solo demandante y que este lo comparta con el resto para sus diferentes usos.

\section{La figura 1 representa el funcionamiento propuesto para el SIES:}

Figura $1^{\text {iv }}$

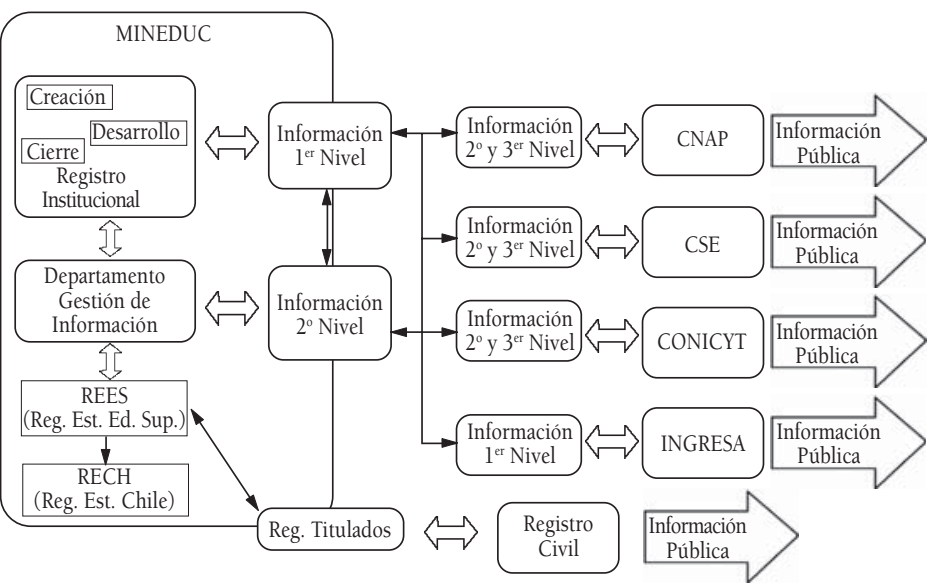

iv Esquema de funcionamiento del SIES: Los elementos representados por círculos son las entidades que conformarían el sistema. Los rectángulos grafican los subsistemas de información y bases de datos que manejan estos organismos. Dentro de ellos se han diferenciado tres niveles de información según el grado de especificidad de los datos que procesan. Las flechas en negro muestran la interacción entre las bases y sistemas para que la información fluya y no se procesen valores diferentes para un mismo dato. Las flechas en blanco señalan las múltiples vías de difusión hacia el público que se pueden dar a partir de datos comunes. 


\section{2. Áreas de información}

\section{Propuesta del conjunto de datos}

Se propone reunir una serie de datos e indicadores elementales para satisfacer la primera línea de información pública, es decir, para postular a algún programa, para responder requisitos básicos y para estudios o caracterizaciones mínimas del sistema de ES.

Las áreas de información fueron clasificadas en siete agrupaciones que recogen los elementos más representativos y de interés para divulgar del sistema de ES. De ellos, el de información pública es el más relevante, por cuanto juega un rol esencial en el encuentro entre la oferta y la demanda de este tipo de enseñanza; es un referente de primera línea sobre las instituciones y un elemento imprescindible del SNAC.

El principal reto es ofrecer datos a los diferentes usuarios en forma oportuna y veraz. Conjugar estos dos elementos es uno de los desafíos principales de esta propuesta, porque no existen los mecanismos o las facultades de un organismo central que obligue a las instituciones a facilitar información con las características señaladas. Es más, existe la tendencia a entregarla parcialmente, con calidad dudosa y retardada lo más posible, ya que puede ser usada como herramienta de competencia entre las instituciones.

Las siete áreas de información son:

\subsection{Información pública}

Se propone crear una canasta de insumos mínimos, necesarios para una buena toma de decisiones por parte del público. Ésta debe ser complementada con información específica de interés particular, entregada por las propias instituciones $u$ otros organismos especializados.

El público objetivo lo componen los estudiantes próximos a ingresar o que cursan ES, sus padres y algunas entidades que actúan 
como intermediarios entre las instituciones y sus clientes (medios de comunicación en general).

Lo que buscan estos usuarios es decidir informadamente en qué programas de ES matricularse y dónde y bajo qué condiciones van a realizar sus estudios. Un objetivo secundario es contrastar la gran cantidad de información que ofrecen las instituciones con algún ente de carácter "oficial" que la valide.

Para identificar las variables más importantes en el ámbito de la demanda de información pública se recurrió a la experiencia acumulada en el Mineduc ${ }^{v}$ y al trabajo investigativo que realizó Dagmar Raczynski y Andrea Canales.

\section{Clasificación de la demanda de información pública}

- Sobre carreras e instituciones: se busca identificar la oferta académica y sus principales características asociadas.

- Sobre ayudas estudiantiles: creciente debido al aumento de la cobertura de matrícula, especialmente en aquellos sectores económicos que requieren apoyo para financiar sus aranceles.

- Sobre servicios: Tales como el pase escolar o inscripción a la Prueba de Selección Universitaria (PSU), reclamos y solicitudes de certificados e informaciones específicas para estudios o investigación.

\subsection{Información sobre docencia de pregrado}

Este ámbito comprende la mayoría de los datos útiles para la difusión pública y el funcionamiento de otros sistemas de gran importancia para la ES, como son el de ayudas estudiantiles o el de asignación de aportes presupuestarios. Desarrollar adecuadamente este sector asegura el éxito del sistema completo.

\footnotetext{
v El trabajo de recopilación y depuración del registro de consultas efectuadas al Mineduc abarcó una serie de datos desde septiembre del año 2002 a octubre del 2004. Esta recopilación sólo fue posible gracias al excelente trabajo realizado por Francesca Hanshing Urra, de la División de Educación Superior.
} 
Como solución para disponer de información precisa sobre los principales indicadores de pregrado se propone la creación de un registro de estudiantes de educación superior. El Registro de Estudiantes de Educación Superior (REES).

La creación del REES responde a la necesidad de contar con mejor, mayor y más oportuna información del sistema, con menores esfuerzos en el largo plazo.

Desde 2002 existe en el Mineduc el Registro de Estudiantes de Chile (RECH), consistente en una base de datos actualizada con los principales datos personales y académicos de todos los estudiantes chilenos de enseñanza básica y media. También, y gracias a un convenio de cooperación con el Registro Civil, el RECH cuenta no sólo con los datos validados de sus estudiantes, sino también de sus padres.

El registro ${ }^{\text {vi }}$ ha mejorado en forma directa la gestión propia de procesos como el pago de subvenciones, el control de estadísticas, la focalización de recursos, entre otros. En forma indirecta, ha apoyado a procesos tales como la inscripción on line para la PSU del año 2004.

El REES se podría implementar mediante el envío de las matrículas de las instituciones con el Rol Único Nacional (RUN) de sus matriculados. Con sólo esto el SIES podría disponer de gran cantidad de información y a muy bajo costo para las propias organizaciones. Sería la continuidad lógica del RECH, pudiéndose enriquecer con otras bases como las de los sistemas de admisión, los de ayudas estudiantiles, pases escolares y de las propias instituciones de ES, quienes de oferentes de datos pasarían también a ser demandantes de esta valiosa información.

vi Abarca García, María Gloria; Barrios Cáceres, José Miguel; Del Río Anabalón, Jorge; Serrano Pacheco, Juan Antonio, "Registro de estudiantes de Chile: un sistema de información masiva en extranet" preparado para el VII Simposio Internacional de Informática Educativa, Leiria, Portugal 2005. Disponible en: http://www.educarchile.cl/ ntg/gestion_escolar/1621/articles-101203_recurso_1.pdf 
El nuevo registro permitiría el manejo certero de asuntos como deserción, movilidad estudiantil, eficiencia de los sistemas de ayuda a los estudiantes, demanda estudiantil, efectividad de los procesos de selección, asignación de recursos, manejo certero del capital humano, entre otras muchas potencialidades, todo con una muy buena relación entre el beneficio y su costo.

\subsection{Docencia de posgrado y postítulo}

Si bien la docencia de posgrado es diferente de la de pregrado para los efectos del SIES, lo más recomendable es replicar la estructura de datos dispuesta para éste con algunas adecuaciones que permitan recoger las especificidades de este ámbito académico.

\subsection{Investigación y generación de conocimiento}

La información sobre este tema tiene que ver, básicamente, con la producción del conocimiento por parte de la institución, en especial, a través de proyectos de investigación y de la publicación del resultado de éstos.

Las variables más utilizadas para informar sobre investigación y difusión provienen de fuentes confiables y con datos comprobables; por ello se privilegian antecedentes sobre concursos y asignaciones provenientes del Estado, o de organismos internacionales, por sobre los provenientes de las propias instituciones.

En cuanto a las publicaciones, se consideran las indexadas en alguna base de datos de prestigio, como Thomson, ISI o SciELO. Desafortunadamente, la indexación en ISI no siempre es todo lo exacta que se piensa, ya que por la forma de afiliación institucional pueden perderse registros de publicaciones.

Para mejorar esta información habría que difundir las definiciones y procesos utilizados para capturar los datos, y destinar recursos para que los encargados de la administración de esa parte del sistema puedan cumplir adecuadamente con esa labor. También 
se debería mejorar el acceso a las mismas fuentes donde se publican los artículos, privilegio de sólo un grupo de instituciones.

\subsection{Vinculación con el medio}

Este ámbito del SIES es el menos desarrollado, ya que las universidades no tienen una forma común de realizar este tipo de actividades, lo cual hace muy difícil diseñar una estructura de datos para todas ellas.

Estudios sobre universidades regionales dan cuenta de la gran cantidad de actividades que desarrollan con su entorno. Eso reafirma que falta mucho trabajo y tiempo para que decanten, con la precisión requerida, el contenido y forma de registrar información respecto de la vinculación.

La propuesta es dejar este punto del SIES como última etapa de desarrollo, a la espera de que la evolución de la ES entregue mayores certezas sobre el tema.

\subsection{Gestión institucional}

Para cada institución sometida a acreditación, el SIES propone registrar el informe FODA ${ }^{\text {vi }}$ de los evaluadores y los desafíos expresados para la siguiente revisión de su acreditación. Para sistematizar esta información se debería recurrir a la pauta que usan los evaluadores, determinando así los campos de información y sus posibles valores.

La propuesta del sistema de información debe contener, por lo menos, datos del balance y estado de resultado, así como de la propiedad de la institución y del uso de recursos públicos entregados a cada universidad.

vii Elemento de la planificación estratégica usada por los pares evaluadores para listar fortalezas y debilidades de los programas o instituciones. 
Lo importante es usar un formato común y bien definido para que la información sea comparable en el tiempo y entre universidades.

\subsection{Gestión interna del sistema}

El SNAC requerirá un número importante de evaluadores, tanto de programas como de instituciones, inscritos en un registro de antecedentes actualizado y disponible para las instituciones. Éste deberá considerar los elementos dispuestos en la ley, así como las participaciones de los evaluadores en los diferentes procesos de acreditación en los que colaboren y otros antecedentes que pueden ser útiles.

Incluir este registro en el SIES permitiría disponer del historial de programas y de instituciones que se presentan al sistema de acreditación; contemplaría los resultados de los procesos, las principales conclusiones y acuerdos, las objeciones y resoluciones, los compromisos o desafíos para revisar en los siguientes ciclos de acreditación, entre otra información de tipo cualitativo o descriptivo.

Un desafío para la operación del SNAC es la actualización de la información relevante de las instituciones y de sus programas durante los periodos de latencia, generados entre cada ciclo de acreditación. La ley establece que las universidades deben actualizar su información ante el organismo encargado de este proceso. La naturaleza propia de las instituciones hará que esto se cumpla parcialmente y lo más probable es que se produzca sólo ante la inminente nueva evaluación. La propuesta para mitigar este comportamiento es que de la captura de datos desde otras entidades debe fluir estos nuevos antecedentes en forma automática, sin que sea necesario para las universidades informar cada cambio ante varios organismos distintos. Como ejemplo, un cambio de autoridad en una universidad se informará al Mineduc y este, al ingresarlo en su sistema automáticamente, lo compartirá con otros organismos para los cuales esta información resulte pertinente. 
Según lo expresado en el Proyecto de Ley, será responsabilidad de la comisión a cargo de la acreditación de programas e instituciones mantener informada adecuadamente a la comunidad respecto de sus decisiones más relevantes. También, disponer de un registro con todos los programas del área de la salud acreditados. Estas tareas se podrán cumplir más fácilmente en tanto se disponga de un sistema de información de gestión interna de los procesos de acreditación como los aquí propuestos.

\section{Variables que reflejan las áreas de información}

Corresponde entonces elaborar, para cada una de las áreas expuestas en el punto anterior, un conjunto de datos e indicadores que reflejen de manera más fiel la evolución que presentan las instituciones.

Es recomendable diseñar un documento, a modo de "diccionario de datos", donde se recoja la definición exacta de cada elemento, quién es responsable de su elaboración y otros detalles de relevancia. El resultado debe ser un documento con determinaciones precisas que orienten la funcionalidad del SIES $S^{\text {viii. }}$.

\section{Canales de difusión}

\section{Oportunidad de entregar esta información}

Es indispensable conjugar el momento más oportuno para entregar la información a los postulantes y los procesos internos de las instituciones que determinan datos como vacantes y precios. Este equilibrio se logra en la víspera de la entrega de los resultados de la PSU.

viii En el trabajo realizado por $\mathrm{CPU}$, referido anteriormente, se propone un documento con estas características y se detallan alrededor de 50 variables como ejemplos para distintas áreas de información. 


\section{Difusión de la información pública}

Tradicionalmente se han usado medios escritos, tales como publicaciones o suplementos que acompañan ediciones de prensa con cobertura nacional. A estos canales de difusión se suman, cada vez más, portales en Internet, que poseen la ventaja de mantener actualizada la información, ofrecer métodos de búsqueda más expeditos, estar asociados a otros portales que complementan la información y ser el medio de comunicación más usado por los jóvenes.

Al canalizar mediante Internet se debe tener en cuenta la dispersión de las fuentes que proveen la información. Por ello, es aconsejable diseñar un portal, sustentado en un modelo de web service, con los entes encargados de cada parte del sistema de información.

Se propone la creación de los siguientes portales web:

- Portal de ayudas estudiantiles: debe contener todos los programas que se ofrecen, tanto para pregrado como para posgrado.

- Portal de actualización de información: restringido a las instituciones y entidades que integran el sistema, es la herramienta para la solicitud y envío de datos pertinentes del sistema.

- Portal de empleabilidad: es la natural evolución del actual sitio "Futuro Laboral", el cual debiera completar sus datos, cubriendo, al menos, la totalidad de instituciones y programas acreditados.

- Portal de atención de público: apoyo, información y gestión de consultas, requerimientos y reclamos del público con relación a las entidades de ES. Debe servir para la tramitación de certificados y otros trámites, tales como el pase escolar.

La integración de estos portales en el portal de la educación superior, donde se adicionen otros servicios, informaciones y direccionamientos, podría ser de gran ayuda para descomprimir solicitudes de intervención del Mineduc ante situaciones entre el público y las instituciones. Es decir, con información y buenos 
servicios se puede dar respuestas a variados requerimientos sin tener que crear una gran infraestructura del tipo de una Superintendencia de Educación Superior.

\section{Bibliografía}

Abarca García, María Gloria; Barrios Cáceres, José Miguel; Del Río Anabalón, Jorge; Serrano Pacheco, Juan Antonio. (2005) Registro de estudiantes de Chile: un sistema de información masiva en extranet. Preparado para el VII Simposio Internacional de Informática Educativa, Leiria, Portugal. Disponible en: http://www.educarchile.cl/ntg/ gestion_escolar/1621/articles-101203_recurso_1.pdf

Peñafiel, Antonio. (2006) Un Sistema de Información para la Educación Superior Chilena. Documento de trabajo $\mathrm{N}^{\circ} 1$ de la Corporación de Promoción Universitaria.

Raczynski, Dagmar y Canales, Andrea. (2000) Demanda de Información sobre Carreras de Pregrado en la Educación Superior Chilena. Asesoría para CIEPLAN. Disponible en: http://www.cnap.cl/estudios/sistema/ 14_raczynski_canales.pdf

Recibido: 8 de mayo de 2006 Aceptado: 30 de mayo de 2006 